\title{
A IMPORTÂNCIA DA ATUAÇÃO DO PSICÓLOGO JUNTO A PACIENTES COM CÂNCER: UMA ABORDAGEM PSICO- ONCOLÓGICA
}

DOI: 10.22289/2446-922X.V2EEA5

\author{
Renata da Fonseca ${ }^{5}$ \\ Marcelo Matta de Castro
}

\begin{abstract}
RESUMO
O câncer é uma doença que tem se destacado pelo aumento no número de casos em todo o mundo. Para esta doença, além da assistência médica, é de fundamental importância uma assistência psicológica adequada. A psico-oncologia surgiu a partir da necessidade do acompanhamento psicológico ao paciente oncológico, além de sua família e toda a equipe que o acompanha. Assim, o papel do psicólogo dentro do contexto oncológico é de prestar apoio psicossocial e psicoterapêutico diante do impacto do diagnóstico das consequências da doença, mostrando possibilidade de auxílio para um melhor enfrentamento e qualidade de vida do doente e de seus familiares. Este estudo teve como objetivo verificar as principais formas de intervenção do Psicólogo no contexto oncológico, bem como a eficácia destas intervenções e os consequentes benefícios desta abordagem para o paciente e seus familiares. Para tanto, foi realizada uma pesquisa bibliográfica, com o levantamento de estudos sobre o tema. A partir deste levantamento, pode-se concluir que no âmbito da oncologia, considerando o sofrimento provocado pela doença, o psicólogo insere-se como um profissional de fundamental importância na composição da equipe multiprofissional, atuando de forma a propiciar uma melhoria na qualidade de vida dos envolvidos neste processo, facilitando o estabelecimento de estratégias de enfrentamento e superação frente ao câncer. Palavras Chave: Psico-oncologia; Assistência Hospitalar; Técnicas de Intervenção.
\end{abstract}

\section{ABSTRACT}

The cancer is a disease that has been standing out by the increase in the number of cases all over the world. For this disease, besides the medical care, is of fundamental importance a suitable psychological assistance. Psico-oncology came from the need to psychological accompaniment to the patient oncologic, besides its family and all the team that accompanies it. This way, the psychologist's paper inside the context oncologic is to render support psychosocial and psychotherapeutic in front of consequences diagnosis impact of the disease, showing help possibility for a better patient life facing and quality and of your family. This study had as goal verify the psychologist intervention main forms in the context oncologic, as well as the effectiveness these interventions and resulting benefits of this approach for the patient and your family. For so much, it was accomplished a bibliographical research, with the studies rising on the theme. From this rising on, it can conclude that in the oncology scope, considering the suffering provoked by the disease, the psychologist it inserts as a fundamental importance

${ }^{5}$ Endereço eletrônico de contato: renatafonseca775@hotmail.com 
professional in the team multiprofessional composition, acting of form to propitiate an improvement in life quality of the involved in this process, facilitating the facing and overcoming front strategies establishment to the cancer.

Key Words: Psycho-Oncology; Psychological Assistance; Psychological Intervention.

\section{INTRODUÇÃO}

O câncer é uma patologia que tem se destacado no campo da saúde pública em todo o mundo na atualidade. Esta patologia compreende um conjunto de mais de cem doenças, que se caracterizam pelo crescimento desordenado de células que invadem os tecidos e órgãos, podendo espalhar-se por outras regiões do corpo. ${ }^{(1)}$

Esse grupo de patologias pode se apresentar sob diversas formas, podendo acometer as mais distintas áreas do corpo, tal como ossos, músculos e demais órgãos internos e externos. ${ }^{(2)}$

É uma doença que tem apresentado altas taxas de prevalência entre pessoas de ambos os sexos e em todas as faixas etárias e, por essa razão tem representado uma das principais causas de mortalidade em todo o mundo. ${ }^{(3)}$

Os avanços nas pesquisas médicas, sobretudo na última década, fez com que muitos tipos de câncer pudessem ser curados, principalmente quando diagnosticados de forma precoce. ${ }^{(4)}$ Ainda assim, o câncer é uma doença que está fortemente associada a ideia de fatalidade, além dos problemas ligados às mudanças físicas, imagem corporal e outras crenças que são adquiridas ao longo do tratamento desta doença. ${ }^{(5)}$

Por tratar-se de uma doença crônica, com prognóstico pouco favorável em muitos casos, o diagnóstico do câncer traz consigo uma série de reações psicológicas negativas tanto nos pacientes como em seus familiares, principalmente ao relacionar a doença com a possibilidade de morte e invalidez. ${ }^{(6)}$

Devido ao impacto psicológico provocado pela doença, tem sido desenvolvidas novas formas de intervenção dentro da Psicologia, a qual se apresenta "[...] com o objetivo de informar, tratar, identificar fatores estressores que podem influenciar no processo de tratamento, bem como planejar de acordo com as necessidades psicossociais do paciente, família e equipe de saúde."(7)

Esse segmento da Psicologia, denominado Psico-oncologia, tem tido maior representatividade nos serviços de saúde em todo o mundo, seja nos hospitais e clínicas de 
oncologia, centros de apoio e tratamento oncológico e demais locais de assistência oncológica.

Diante deste contexto, o presente estudo teve como objetivo verificar as principais formas de intervenção do Psicólogo no contexto oncológico, bem como a eficácia destas intervenções. Pretende-se ainda, a partir deste estudo, verificar a importância da atuação do Psicólogo na assistência oncológica, bem como os benefícios percebidos pelo paciente e pela família a partir da atuação deste profissional.

Para atingir aos objetivos propostos no presente estudo, foi realizado um levantamento bibliográfico de textos publicados preferencialmente de 2001 a 2015, em três importantes bases de dados brasileiras de publicações eletrônicas, a saber: Index Psi (Conselho Federal de Psicologia/PUC-Campinas), PEPsic (Periódicos eletrônicos em Psicologia) e Scielo Brasil, bem como ao acervo bibliográfico sobre o tema, a fim de se levantar as principais teorias concernentes ao tema estudado.

Os termos de pesquisa utilizados para a realização do levantamento do material de estudo foram: psico-oncologia; abordagem psicológica em pacientes oncológicos; psicologia e câncer; psicologia oncológica; e intervenção psicológica em pacientes oncológicos.

Numa análise preliminar, foram excluídos todos os textos que não evidenciaram algum tipo de relação ou interface com o tema de estudo (intervenção em psico-oncologia) e também aquelas que, embora resgatadas pelos descritores utilizados, não se apresentaram coerentes aos objetivos da pesquisa.

Também se utilizou como critério de seleção os artigos cujo texto completo encontrava-se disponível nas bases de dados. Após a seleção dos textos, foi feita análise, fichamento e, posteriormente, os dados compilados foram utilizados na produção do presente estudo.

\section{CARACTERIZAÇÃO DO CÂNCER}

Câncer é o conjunto de doenças que tem como característica o crescimento desordenado e difuso de células anormais, causado por fatores externos como a exposição a produtos químicos, tabaco, radiações e infecções por organismos) ou também internos (mutações herdadas ou que podem ocorrer ao longo do metabolismo, devido a hormônios e ainda por condições imunológicas). Tais fatores Outubro, 2016:2(Edição Especial): 54-72. 
podem atuar juntos ou de forma sequencial, culminando com o início do processo da carcinogênse. ${ }^{(8)}$

A palavra câncer tem origem no termo latino cancer, que significa 'caranguejo'. Supõe-se que essa palavra foi utilizada em analogia ao modo infiltrante de crescimento da doença, que se assemelha às pernas deste crustáceo. ${ }^{(9)}$

Esta doença se caracteriza pela multiplicação e propagação descontrolada de formas anômalas das próprias células do organismo. No câncer, as células cancerosas apresentam características específicas que as distinguem das células normais do organismo, tais como a capacidade de diferenciação e perda de função, proliferação descontrolada, poder invasivo e capacidade de formar metástase. ${ }^{(10)}$

A proliferação de células cancerosas não encontra-se sujeita ao controle pelos processos que regulam a divisão celular normal e o crescimento dos tecidos. Tal aspecto é, portanto, aquele que mais distingue as células cancerígenas das células normais, ou seja, a proliferação de células responsáveis pela doença não encontra-se sujeita aos processos reguladores normais do organismo. ${ }^{(10)}$

Os fatores de risco relacionados a ocorrência do câncer podem ser encontrados no meio ambiente ou ainda sofrer influência de aspectos hereditários. $A$ maioria dos casos encontram-se relacionados ao meio ambiente, com a influência de um grande número de fatores de risco. Desta forma, as mudanças provocadas no meio ambiente aliada aos hábitos e estilos de vida do homem moderno, podem influenciar de forma substancial na indução de diversos tipos de câncer.(9)

É importante destacar queo câncer é uma patologia cujo tratamento é, geralmente, bastante doloroso, com efeitos colaterais que desencadeiam mudanças na vida do indivíduo. Em muitos casos, o indivíduo portador da doença perde sua independência, sofre com alterações em sua imagem corporal, se isola de seus vínculos sociais, se afasta de atividades de lazer e apresenta sentimento de inutilidade, podendo levar ao surgimento de diversos distúrbios psicológicos. ${ }^{(11)}$

\subsection{ASPECTOS PSICOLÓGICOS DA DOENÇA}

O processo de adoecimento, sobretudo nos casos em que existe a eminência de morte, é caracterizado por problemas emocionais e pelo sofrimento 
psicossocial do doente. Quando se trata do câncer, tais aspectos podem ser intensificados em virtude das características da doença.(12)

Diante do diagnóstico de câncer ocorrem diversas mudanças na vida do sujeito, com a ameaça de planejamentos futuros, perda da esperança, ansiedade e medo. O diagnóstico da doença passa então a ser encarado como o início de uma crise, que se implanta em meio a muitas incertezas e inseguranças. ${ }^{(13)}$

O impacto provocado pelo adoecimento poderá depender de diversos fatores, entre eles, a cronicidade da doença, seu prognóstico, sentimentos de ameaça, ou ainda devido ao temor de que essa doença possa lhe trazer limitações ou dificuldades. ${ }^{(14)}$

Ao ser diagnosticado com a doença, o paciente passa a se confrontar com questões ligadas à morte, o que poder fazer gerar nele um sentimento de vulnerabilidade e perda de controle sobre a própria vida. ${ }^{(15)} \mathrm{Em}$ casos de doenças graves, incluindo-se o câncer, as pessoas podem adotar estratégias de enfrentamento que podem ser classificadas como centradas no problema ou na emoção. Quando elas são classificadas no grupo de centradas no problema, o paciente busca administrar ou até mesmo alterar o problema ou o seu relacionamento com o meio. Tais estratégias ganham um caráter adaptativo, voltadas para a realidade, buscando uma remoção ou mesmo uma tentativa da abrandar a fonte estressora. ${ }^{(16)}$

No caso do grupo que encontra-se centrados na emoção, as estratégias de enfrentamento tentam substituir ou regular o impacto emocional que a doença provoca. Deste modo, surgem processos defensivos fazendo com que os pacientes procurem não confrontar conscientemente com o seu problema e a sua realidade ameaçada. ${ }^{(16)}$

\section{AS PRINCIPAIS INTERVENÇÕES CLÍNICAS DO PSICÓLOGO NA ASSISTÊNCIA A PACIENTES EM TRATAMENTO ONCOLÓGICO}

Diferentes acontecimentos e eventos nas ciências levaram a um campo bastante fértil para o desenvolvimento da psicologia, da psiquiatria e da medicina em todo o mundo.(17)

Tratando-se da inserção do psicólogoem contextos de assistência a pacientes hospitalizados, verifica-se que após diversas revisões e questionamentos quanto a sua Outubro, 2016:2(Edição Especial): 54-72. 
aplicação, ela se estabeleceu como uma importante alternativa na busca pela compreensão da existência humana e suas respectivas contribuições para os pacientes submetidos a experiência da hospitalização ${ }^{(18)}$

Logo, no âmbito hospitalar a Psicologia busca, através de sua intervenção, minimizar o sofrimento provocado no paciente em virtude do processo de internação hospitalar, além de prevenir possíveis sequelas e implicações emocionais futuras provocadas por esse período. ${ }^{(18)}$

A atuação da Psicologia especificamente no tratamento dos pacientes oncológicos teve seu início a partir da década de 70 em virtude dos vários aspectos psicológicos que se encontram relacionados ao paciente portador de câncer. Assim, o surgimento deste segmento somente foi possível com a diminuição do estigma existente em relação a esta doença, o que permitiu importantes mudanças de atitudes em relação ao câncer e ao seu portador. ${ }^{(19)}$

O estigma e os mitos que envolviam o câncer puderam ser substituídos por conhecimento e instrumentos de enfrentamento tais como os tratamentos, grupos de autoajuda, suporte psicossocial etc. A partir desses fatos foi possível explorar e estudar respostas e comportamentos psicológicos associados ao câncer. ${ }^{(17)}$

No início do século $X X$, o desenvolvimento de técnicas cirúrgicas e das medicações anestésicas propiciaram uma melhoria no tratamento do câncer, mas sempre na dependência do tipo de tumor e da fase em que era descoberto.(19)

$\mathrm{Na}$ verdade, o que se pode observar é a atualidade da Psico-Oncologia enquanto área que propicia a união de diferentes profissionais promotores de saúde, respondendo portanto a uma tendência mundial no sentido de formar profissionais que atuem em conjunto, contribuindo com suas diferentes visões para o enriquecimento do cuidado com os pacientes.

\subsection{UMA ABORDAGEM PSICOLÓGICA NO CÂNCER: A PSICO-ONCOLOGIA}

O termo Psico-Oncologia foi proposto pela primeira vez pelo cirurgião oncológico e psicanalista argentino José Schavèlson no ano de 1961. Ele usou este termo para designar essa nova área do conhecimento, formada por um ramo da medicina que se ocuparia em prestar assistência ao paciente com câncer, do seu contexto familiar e social e dos aspectos médico-administrativos presentes no cotidiano do paciente com câncer. ${ }^{(20)}$

A Psico-Oncologia pode ser definida como uma especialidade que se propõe em estudar duas dimensões psicológicas presentes no diagnóstico do câncer, o impacto dessa doença no funcionamento emocional do paciente, de sua família e dos profissionais 
envolvidos em seu tratamento e também o papel das variáveis psicológicas e comportamentais na incidência e sobrevivência do câncer. ${ }^{(19)}$

A Psico-Oncologia representa uma área do conhecimento da Psicologia da Saúde, tratando dos cuidados com o paciente oncológico, bem como sua família e os profissionais que se encontram envolvidos no seu tratamento. Logo, a Psico-oncologia é uma área voltada para as questões emocionais do paciente portador de câncer. ${ }^{(20)}$

Apesar dos avanços em relação ao conhecimento em Psico-Oncologia, até o início dos anos 80 , investigações detalhadas acerca da influência de fatores de natureza psicológica sobre o desenvolvimento do câncer não representavam uma rotina nos centros médicos especializados em oncologia. Também não se estudava de forma satisfatória o impacto de variáveis psicossociais, consideradas isoladamente ou em conjunto, sobre o tratamento do câncer. ${ }^{(21)}$

A partir do conhecimento já adquirido até hoje em relação a Psico-Oncologia, já é possível encontrar a definição de circunstâncias em que é indicada a necessidade de ajuda psicológica ao paciente oncológico. A maioria delas encontra-se fundamentada na identificação de variáveis de natureza psicossociais do paciente e seu familiar, tal como ocorrem nas reações emocionais do paciente ou da família, impedindo-o de buscar tratamento e cooperar com o tratamento em andamento, ou interferindo de forma negativa sobre o processo de tratamento. (21)

Em relação às variáveis psicossociais, a assistência ao paciente com câncer sua respectiva família, deveria envolver a compreensão da interação entre diversos fatores: desenvolvimentais, considerados os diferentes contextos de desenvolvimento; socioeconômicos e culturais do paciente e dos familiares; capacidade de enfrentamento de situações estressantes; nível de coesão e facilidade de comunicação entre os membros da família; história pessoal e familiar do paciente. A compreensão mais precisa da influência destes fatores poderia ajudar a delimitar, inclusive metodologicamente, a intervenção profissional da Psicologia dentro da Oncologia. (22)

Os estudos em Psico-Oncologia compreendem muito além da identificação de variáveis de risco psicossocial ou da delimitação de circunstâncias em que é indicada a necessidade de ajuda psicológica ao paciente. É de suma importância um planejamento ambiental orientado ao desenvolvimento comportamental do paciente. A delimitação de circunstâncias específicas ou a definição de variáveis psicossociais isoladas não são suficientes, é necessário entender a relação funcional entre o paciente e o ambiente em que são dispensados os cuidados com o tratamento (hospitalar, doméstico ou outro). ${ }^{(23)}$

Um aspecto que vem sendo abordado pela Psico-Oncologia é a necessidade do paciente saber de sua doença e de seu estado, várias são as discussões sobre a comunicação 
do diagnóstico. Diferentemente do que acontece em países desenvolvidos, como no caso dos Estados Unidos e de países da Europa, observa-se uma grande dificuldade de comunicar o diagnóstico de câncer aqui no Brasil. É importante que o profissional saiba como contar e transmitir a certeza que o paciente receberá o melhor tratamento, e que a cura é uma meta em conjunto. ${ }^{(4)}$ Diversos estudiosos da Psico-oncologia discutem esta questão da informação do diagnóstico sobre o que contar, como contar e quem contar. Parece haver um consenso de que se deve informar o paciente de acordo com o que ele deseja e está preparado para saber, sendo assim, ele sinaliza o quanto ele pode e deseja saber.(2)

Destaca-se a importância da aquisição de conhecimentos relativos ao câncer e ao tratamento para o enfrentamento efetivo. Isso contribui em tomadas de decisões do paciente acerca do seu tratamento, informando adequadamente familiares e amigos, planejando o futuro e, dessa forma, criando um maior controle da situação. Somado a isto, intervenções voltadas para a reconstrução de representações sobre a doença e a terapêutica podem ajudar no manejo da ansiedade, uma vez que o problema passa a ser considerado de forma diferente, clara e organizada, trabalhando os sentimentos relacionados à doença - dentre eles, o medo da morte, que pode ser aterrorizante. ${ }^{(11)}$

No Brasil o progressivo avanço da Psico-oncologia no que se refere ao aumento da demanda por atendimento psicológico nos casos ligados ao câncer. Neste contexto, ela se define como uma área de atuação interdisciplinar, realizada através de uma equipe integrada, de acordo com uma visão abrangente que vai além da doença, contemplando o paciente e todo o meio que o cerca. ${ }^{(20)}$

Apesar dos avanços no campo das Ciências Médicas em relação ao câncer, muitos ainda são os desafios impostos pela doença à sociedade, muitos também são os conhecimentos e novas práticas que emergem na busca por melhorias no atendimento aos envolvidos nesta importante questão de saúde pública. ${ }^{(24)}$

A Psico-Oncologia não apresenta uma proposta teórica definida, entretanto ela busca um 'Modelo Teórico de Intervenção', que tem como objetivo o atendimento integral do paciente, do seu familiar e das equipes de saúde. ${ }^{(17)}$

Atualmente, o eixo das pesquisas e intervenções em Psico-Oncologia tem sofrido forte ampliação, principalmente porque tem se destinado não apenas a questão dos sobreviventes da doença, mas também destinado suas intervenções aos cuidadores de pacientes oncológicos, seja eles formais (profissionais) ou informais (familiares), visando melhorar a sua qualidade de vida no trabalho. ${ }^{(25)}$

\subsection{A PRÁTICA PSICOLÓGICA EM PACIENTES ONCOLÓGICOS}

Outubro, 2016:2(Edição Especial): 54-72. 
O indivíduo com câncer necessita lidar com mudanças emocionais, sociais e físicas que acompanham a doença e seus tratamentos. A forma como se adaptará nos aspectos cognitivos e comportamentais em relação a tais mudanças sofrerá influência de diversos aspectos, variando bastante entre os pacientes. Muito tem sido investido no conhecimento e identificação das variáveis que influenciam no processo de ajustamento ao câncer, levando-se em conta a perspectiva biopsicossocial do processo saúde doença. Os estudos sobre as dimensões psicossociais relacionadas à adaptação ao câncer e dos aspectos que facilitam ou dificultam o processo de ajustamento, culminaram na busca de fatores que preveem a adaptação. (22)

Apesar da falta de consistência entre os estudos sobre quais variáveis são mais influentes, o ajustamento psicológico tem sido um importante conceito pesquisado por se referir a forma como o paciente lida com as ameaças específicas do câncer e seus tratamentos. O ajustamento ao câncer é o processo de aprender e se adaptar às diversas mudanças vivenciadas como resultado do adoecimento e tratamento. Para o autor, o grau de ajustamento dependerá do efeito combinado das respostas de enfrentamento, suporte social e da avaliação cognitiva que o indivíduo faz sobre sua experiência. Além dessas variáveis, é comum autores relacionarem o senso de significado na vida e as condições físicas do paciente à sua adaptação. ${ }^{(23)}$

Em relação à gravidade da doença, ressalta-se que essa não pode ser considerada a única responsável por reações desadaptativas. Estudos identificaram prejuízos à adaptação psicológica ao câncer mesmo quando esse é potencialmente curável, indicando que pacientes com bom prognóstico também apresentam redução na qualidade de vida, dificuldades afetivas e cognitivas, elevada ansiedade e prejuízos no ajustamento psicológico. ${ }^{(26)}$

A necessidade de internação também é apontada como condição que aumenta o nível de stress e desencadeia desordens mentais para os pacientes com câncer. Entretanto, não verificaram as características das pacientes envolvidas no processo de ajustamento ao contexto de internação, considerando apenas esse fator como indicador de risco. ${ }^{(23)}$

Depara-se, muitas vezes, na literatura com estudos que consideram oestressor, por si só, um indicador de risco de dificuldades psicológicas. Tal abordagem acaba não auxiliando na compreensão dos fatores envolvidos na forma como o paciente lidará com o estressor, desviando a atenção dos aspectos que contribuem para aumentar ou diminuir a vulnerabilidade em determinadas situações. Identificar e conhecer a capacidade estressora de determinado contexto pode facilitar a elaboração de programas de intervenção psicológica direcionados aos pacientes que serão expostos a ele. Porém, considerar o estressor como 
único indicador de risco não contribui para a compreensão das variáveis implicadas no processo de enfrentamento e ajustamento ao contexto de adoecimento por câncer. ${ }^{(27)}$

Apesar do maior investimento para conhecer as variáveis que interferem no processo de ajustamento, poucas tentativas têm sido realizadas para que as intervenções auxiliem efetivamente os pacientes com fatores de risco para má adaptação psicológica, mantendo-se o foco mais no tratar do que no prevenir. ${ }^{(28)}$

Talvez, aumentar o cuidado com os fatores envolvidos no processo de ajustamento e elaborar estratégias para identificá-los precocemente, contribua para a diminuição do impacto do câncer, possibilitando a estruturação de protocolos de intervenção em psicologia da saúde que ultrapassem o agir apenas quando a dificuldade diante da doença já está instalada. ${ }^{(29)}$

\subsection{EFEITOS ESPERADOS NAS INTERVENÇõES CLÍNICAS DO PSICÓLOGO NO ÂMBITO ONCOLÓGICO}

É sabido que a atuação dos profissionais da psicologia, embora guarde importante relação com a atuação dos profissionais da medicina que atendem ao paciente, com esta não se confunde, tendo o psicólogo uma dimensão mais social e humana. ${ }^{(29)}$

A partir do diagnóstico de uma neoplasia, altera-se a dinâmica da família, bem como sua rotina, de acordo com as personalidades e modos distintos de cada pessoa, se tornando essencial o acompanhamento emocional, principalmente quando a doença ocorre em crianças, nas quais o câncer determina expressões de pena e pesar devido aos seus medos e mitos. ${ }^{(30)}$

Entretanto o profissional que irá atuar em auxílio às famílias enfrenta as diversidades neste tipo de atendimento, sobretudo no que se refere à complicada conceituação de família, principalmente quando se trata da família brasileira. Isso ocorre porque ela se caracteriza por uma pluralidade de composições que se dão por diversos meios como os laços sanguíneos, relação não formalizada por parentesco, família conjugal e extensa, núcleo doméstico e família com união estável, etc. ${ }^{(31)}$

A compreensão das diferenças existentes envolvidas em todo o processo do adoecimento, bem como no ciclo de vida familiar, implica no comprometimento e disposição dos profissionais envolvidos em acompanhar as variadas mudanças e surpresas que a ele podem se apresentar. ${ }^{(31)}$

O doente, juntamente com a família, passa por um período de crise, que se dá desde o diagnóstico, até o esclarecimento da situação e possíveis planos de tratamento. É 
nessa fase que surgirá a necessidade de aprendizados práticos, como lidar com a dor e com o ambiente hospitalar. ${ }^{(29)}$

A família também sofre com a descoberta do câncer porque ela tem a concepção de que os filhos devem necessariamente sobreviver aos seus pais. Assim, o câncer infantil causa um intenso medo na família, acerca de uma possível mudança, no curso dos acontecimentos naturais da vida, o falecimento precoce de um filho. ${ }^{(31)}$

O diagnóstico de um câncer quebra a invulnerabilidade tão presente na sociedade atual, caracterizada pelo seu aspecto capitalista e consumista, valorizando e estimulando o novo, além de possuir um sentimento de inutilidade quanto ao velho e a morte. ${ }^{(31)}$

O câncer faz recair sobre a família do doente uma série de sentimentos, podendo pelas características danosas da doença ser recebido pela família com reações de culpa, raiva, inconformismo, além de depressão e isolamento. A negação da realidade, que pode atingir os familiares e os próprios doentes, aliada a desconfiança quanto à validade do diagnóstico, também podem ocorrer na ocasião do diagnóstico da doença. ${ }^{(32)}$

Assim, os membros da família do paciente podem apresentar sintomas somáticos, que se caracterizam por males físicos ou até mesmo diminuição da atenção e irritabilidade. Essas situações comprometem a capacidade de se lidar com as informações e procedimentos que parecem evidentes para a equipe hospitalar. Neste momento, não basta explicar somente uma vez, mas é necessário que o profissional reitere novamente várias vezes, e acompanhe a possibilidade de aceitação dos familiares. ${ }^{(32)}$

Em algumas circunstâncias específicas, verifica-se com exatidão, a necessidade do suporte psicológico ao paciente. Isso ocorre em casos em que as reações emocionais do paciente dificultem o tratamento a ser realizado. Ele pode agir de forma a não cooperar e a não expressar sentimentos a respeito das reais condições em que se encontra. Poderá ainda reagir de forma a ampliar o medo perante a doença e assim criar resistência a situações, às quais necessita ser submetida, como uma internação. Nesse caso o paciente poderá ter maior percepção da dor ou degradação corporal. A psico-oncologia tem papel fundamental também quando existe a perturbação no desempenho de atividades sociais e de lazer ou quando há o aparecimento de sintomas psiquiátricos como angústia e depressão. (23)

Deve-se ressaltar ainda o papel do psicólogo em sua atuação junto ao paciente oncológico e sua família como promotor de mecanismos de enfrentamento, possibilitando a esses sujeitos enfrentar essa problemática situação de adoecimento como a menor possibilidade possível de disfunções comportamentais. ${ }^{(18)}$

Este processo, conhecido como coping, tem o papel de administrar e veicular estratégias que possibilitem aos envolvidos, seja o próprio paciente como seus respectivos familiares, adotar mecanismos que reduzam, minimizem ou estabeleçam formas de Outubro, 2016:2(Edição Especial): 54-72. 
tolerância, frente a situações estressoras, tais como as observadas com os pacientes oncológicos. ${ }^{(18)}$

O psicólogo deve atuar junto ao paciente oncológico, à família e também à sociedade. Em relação ao paciente oncológico deve: auxiliar no momento do diagnóstico; informar sobre a doença; esclarecer os tratamentos a que será submetido e os possíveis efeitos colaterais e como lidar com eles; ajudar o paciente a enfrentar a doença através de técnicas específicas, visando sua participação ativa na evolução do tratamento. ${ }^{(32)}$

\subsection{AS INTERVENÇÕES EM PSICO-ONCOLOGIA}

As intervenções em Psico-Oncologia não diferem daquelas realizadas na psicologia em geral, onde o objetivo é sempre o bem-estar do paciente.

Os primeiros profissionais ligados à promoção de saúde que começaram o processo de dar alguma assistência de caráter psicológico aos pacientes de câncer foram os enfermeiros e assistentes sociais. ${ }^{(19)}$

Para este autor, em virtude da própria proximidade e convívio com os pacientes, esses profissionais começam a perceber a demanda por algum tipo de atendimento que auxiliasse nas reações emocionais em relação ao progresso da doença e aos tratamentos aos quais eram submetidos. ${ }^{(19)}$

O que distingue o profissional de Psico-Oncologia é que, para prestar assistência a um paciente portador de câncer, seus familiares ou mesmo à equipe de saúde que atende esse tipo de paciente, esse profissional deve dominar alguns aspectos não exigidos necessariamente em outros tipos de atendimento. ${ }^{(33)}$

O que se exige, inicialmente, em relação a atuação em Psico-Oncologia diz respeito a necessidade de conhecimento em relação ao câncer e ainda as principais formas de tratamento a que estes pacientes podem ser submetidos. Não é importante dominar o discurso do oncologista, porém é necessário um conhecimento básico da doença e suas modalidades. A razão dessa necessidade reside no fato de que, para cada tipo de câncer, suas características, sua localização e o processo terapêutico que se submeta, o paciente apresentará um determinado tipo de reação. ${ }^{(34)}$

Mesmo que se saiba que essa reação ocorre, não se pode prever a forma com que cada pessoa irá reagir a ela. A mesma dependerá de algumas variáveis, tais como, a idade do paciente, sua classe socioeconômica, sua estrutura psíquica, sua personalidade em relação a suportar frustrações e a enfrentar os eventos da vida, variáveis estas pré-existentes ao surgimento da doença. ${ }^{(22)}$

Outubro, 2016:2(Edição Especial): 54-72. 
Em relação aos tratamentos, o mais importante é o conhecimento do psicólogo, pois os efeitos colaterais são vários e podem gerar estados emocionaiscomplexos. Uma interpretação inadequada desses estados pode ser prejudicial ao paciente. ${ }^{(35)}$

Um dos principais aspectos a se considerar nos casos dos pacientes oncológicos é a depressão. Sabe-se que um estado depressivo pode afetar o sistema imunológico do paciente, sendo assim, importante que esse sintoma seja detectado o mais precocemente possível para que o paciente possa ser medicado e o problema possa ser eventualmente atenuado ou eliminado. ${ }^{(29)}$

A partir dessas reflexões, fica evidente a necessidade de uma formação específica para atendimento de pacientes portadores de câncer. Isto não significa, porém, que sem essa formação um profissional não possa desenvolver seu trabalho caso se depare com um paciente doente. Só o ato de acolher, ouvir e estar ao lado de uma pessoa doente já é reconfortante para ela. Porém, a cada dia, cresce a procura por cursos de formação em PsicoOncologia, por profissionais que desejam se instrumentar melhor para assistir pacientes portadores de câncer. ${ }^{(36)}$

A partir da Portaria 3.535/98 do Ministério da Saúde, passou a ser obrigatória a presença de um profissional de psicologia em todas as clínicas ou instituições que atendam a pacientes de câncer. ${ }^{(37)}$ Embora essa lei não seja cumprida de modo geral, muitos profissionais foram pegos de surpresa, sendo obrigados a prestar atendimento a pacientes portadores de neoplasia.(2)

Uma das preocupações da Psico-Oncologia consiste no cuidado com os cuidadores. Por cuidador pode-se entender tanto o cuidador profissionalizado, pertencente a uma equipe de promoção de saúde, quanto o cuidador domiciliar, que pode ser um enfermeiro contratado, um familiar, um vizinho, ou seja, aquele que estiver disponível e que o paciente aceite como o que vai cuidar dele.(38)

Neste sentido, os autores afirmam que tanto a equipe no atendimento quanto o cuidador individual do paciente enfrentam situações semelhantes de angústia, desamparo, frustração e impotência, sendo importante que se tenha um olhar sobre suas necessidades e demandas. ${ }^{(38)}$

Considerando os possíveis danos psicológicos advindos da assistência a pacientes oncológicos para os profissionais e cuidadores individuais, deve-se considerar que estes, por encontrarem-se sujeitos ao desenvolvimento de diversos distúrbios psicopatológicos e esgotamento profissional (18), também necessitam, por sua vez, de uma assistência psicológica que garanta a esses indivíduos a manutenção de boas condições psicológicas, evitando-se assim possíveis impactos emocionais decorrentes do cuidado com esses pacientes.

Outubro, 2016:2(Edição Especial): 54-72. 


\section{DISCUSSÃo}

A partir do levantamento bibliográfico realizado e considerando os descritores adotados para a pesquisa, obteve-se, inicialmente, um total de 82 artigos científicos, 19 dissertações/teses e 14 livros acerca do tema.

Com a seleção do material e a utilização dos critérios de exclusão, obteve-se um total de 23 artigos científicos, 04 livros e 03 teses/dissertações que foram utilizados na produção final do estudo.

A partir da análise das obras selecionadas pode-se ressaltar que considerando a importância da presença do psicólogo no processo terapêutico em pacientes oncológicos, aponta em seu estudo que pacientes submetidos ao acompanhamento psicológico durante o tratamento apresenta ganhos significativos em diversos aspectos como a melhora do estado geral de saúde; melhor tolerância aos efeitos adversos da terapêutica oncológica, melhora da qualidade de vida além de proporcionar uma melhor comunicação entre paciente, família e equipe. ${ }^{(29)}$

A partir desta constatação pode-se inferir que a intervenção psicológica, muitos pacientes voltam a ter prazer pela vida, trabalhando suas angústias, fortalecendo suas convicções deque o tratamento é eficiente e de que existem poderosas defesas em seu corpo, desmistificando crenças errôneas, elaborando conflitos existentes e, enfim, facilitando ao paciente a obtenção de uma clara percepção sobre si mesmo. ${ }^{(24)}$

A experiência da Psico-oncologia no Hospital do Câncer de Barretos, demonstra que esta abordagem permite a pacientes e familiares lidar com as transformações e estratégias advindas do adoecimento pelo câncer de maneira mais tranquila e adequada às condições que se encontram. ${ }^{(33)}$

De forma geral, os textos selecionados apresentaram a importância da intervenção psicológica junto aos pacientes portadores do câncer, demonstrando os benefícios obtidos com este tipo de intervenção.

Vale ressaltar que, apesar do elevado número de estudos realizados em relação ao tema, poucos foram os que mensuraram os benefícios e o impacto positivo de uma abordagem Psico-oncológica. Tais trabalhos são de grande importância para uma maior valorização deste profissional enquanto membro de uma equipe de apoio em unidades de atendimento oncológico, seja em nível ambulatorial como hospitalar.

Por fim, pode-se destacar que os maiores desafios ao trabalho do psicólogo no ambiente oncológico é a falta de reconhecimento por muitos médicos, bem como pode contrariar orientações dadas por estes, quando aos aspectos psicológicos dos casos em Outubro, 2016:2(Edição Especial): 54-72. 
atendimento. Algumas vezes são os enfermeiros que se sentem invadidos ou criticados na sua atuação, pelos psicólogos.(2) Porém, deve ser considerado que a chegada da PsicoOncologia no ambiente hospitalar, sobretudo para assistência aos casos de câncer é recente e sua função ainda é frequentemente desconhecida ou distorcida, prejudicando muito a legitimidade deste profissional no contexto da Oncologia.

\section{CONCLUSÃO}

O câncer tem se consolidado no Brasil como uma das principais causas de morte, apresentando ainda números assustadores de novos casos nos últimos anos. Esse crescimento de novos casos, aliado às características desta patologia justifica a necessidade de se estimular e intensificar os cuidados paliativos com esses pacientes, superando assim a assistência médica como única forma de beneficiar os pacientes oncológicos.

Neste sentido, a Psico-Oncologia se destaca como segmento da Psicologia de grande relevância, sobretudo em se considerando o cenário desta doença na contemporaneidade.

Diante do sofrimento que caracteriza o processo de evolução da doença, o Psicólogo torna-se um profissional indispensável, uma vez que este poderá criar um espaço de escuta, acolhimento e reflexão tanto para o paciente como para todo o seu grupo familiar, que também compartilha do sofrimento da doença.

Assim, a partir da análise da produção bibliográfica sobre tema, foi possível verificar que a Psico-Oncologia atua de forma a propiciar uma melhoria na qualidade de vida dos envolvidos neste processo, facilitando o estabelecimento de estratégias de enfrentamento e superação frente ao câncer.

\section{REFERÊNCIAS}

1. INCA. Como surge o Câncer? 2008. Disponível em:<http://www.inca.gov.br/conteudo_view.asp?id=317.>.

2. Carvalho MM. Psico-oncologia: história, características e desafios. Psicol USP [periódico na internet]. 2002 [acesso em 2015 set 29]; 13(1):151-166. Disponível em: $<$ http://www.scielo.br/scielo.php?script=sci_arttext\&pid=S010365642002000100008>. 
3. Martins AM. Relações de Gênero e a Atuação de Psicólogos na Oncologia:Subsídios para a Saúde Masculina. Psico[periódico na internet]. 2014 [acesso em 2015 set 29]; 45(1):7-14. Disponível <http://revistaseletronicas.pucrs.br/ojs/index.php/revistapsico/article/view/11998>.

4. Yamaguchi NH. O câncer na visão da oncologia. In: Carvalho, MMMJ (Org.).Introdução à Psiconcologia. São Paulo: Livro Peno; 2003.

5. Silva VCE. O impacto da revelação do diagnóstico de câncer na percepção do paciente [tese] [periódico na internet]. Ribeirão Preto: Universidade de São Paulo [acesso em 2015 set 28]; Disponível em:<http://www.teses.usp.br/teses/disponiveis/22/22132/tde11052005-112949/.>

6. Castro ESA, Souza AM. Cuidando da pessoa com câncer: contribuições da GestaltTerapia. Rev IGT Rede[periódico na internet]. 2012 [acesso em 2015 out 5]; 9(16):43$69 . \quad$ Disponível em: <http://www.igt.psc.br/ojs/include/getdoc.php?id=2029\&article=367\&mode=pdf >.

7. Froelich TC. Psico-oncologiaeterminalidade:casos em que o paciente é uma criança. JornPesq Psic. [periódico na internet]. 2011 [acesso em 2015 set 21]; 4:1-10. Disponível em: <http://online.unisc.br/acadnet/anais/index.php/jornada_psicologia/article/view/10193 $>$.

8. Cotran RS, Kumar V, Collins, T. Patologia Estrutural e Funcional. Guanabara: Rio de Janeiro; 2000.

9. Almeida VL, et al. Câncer e agentes antineoplásicos ciclo-celular específicos e ciclocelularnão específicos que interagem com o DNA: uma introdução. QuímNova [periódico na internet]. 2005 [acesso em 2015 set 21]; 28(1):118-129. Disponível em: <http://www.scielo.br/pdf/qn/v28n1/23048.pdf>.

10. Rang HP; Dale MM, Ritter JM. Farmacologia. Rio de Janeiro: Guanabara Koogan; 2001.

11. Santana JJRA,Zanim CR, ManigliaJV. Pacientes com câncer:enfrentamento, rede social e apoio social. Paidéia[periódico na internet]. 2008 [acesso em 2015 set 28]; 18(40):372-384. Disponível em: <http://www.scielo.br/pdf/paideia/v18n40/13.pdf>.

12. Cardoso G, Luengo A, Trancas B, Vieira C, Reis D. Aspectos Psicológicos do Doente Oncológico. Rev. Ser. Psiq. Hosp.Prof. Dr Fernando Fonseca [periódico na internet]. 2009 [acesso em 2015 set 28]; 6(2):8-19. Disponível em:

Outubro, 2016:2(Edição Especial): 54-72. 
<http://www.psilogos.com/Revista/Vol6N1/Indice9_ficheiros/Cardoso\%20et\%20al\%2 0_\%20p8-19.pdf>

13. Silva SS, Aquino TAA, Santos RM. O paciente com câncer: cognições e emoções a partir do diagnóstico. RevBras Ter Cogn[periódico na internet]. 2008 [acesso em 2015 set 21]; 4(2):73-88. Disponível em: <http://pepsic.bvsalud.org/scielo.php?script=sci_arttext\&pid=S1808$56872008000200006>$.

14. Nucci NAG. Qualidade de vida e câncer: um estudo compreensivo [tese] [periódico na internet]. Ribeirão Preto: Universidade de São Paulo; 2003 [acesso em 2015 set 28]; Disponível em: <http://www.teses.usp.br/teses/disponiveis/59/59137/tde-27012004$222429 />$.

15. Rossi L, Santos, M. Repercussões psicológicas do adoecimento e tratamento em mulheres acometidas pelo câncer de mama. PsicolCiencProf[periódico na internet]. 2003 [acesso em 2015 set 21]; 23(4):32-41. Disponível em: <http://www.scielo.br/pdf/pcp/v23n4/v23n4a06.pdf>.

16. Lorencetti A, Simonetti JP. As estratégias de enfrentamento de pacientes durante o tratamento deradioterapia.Rev Latino-am Enfermagem[periódico na internet]. 2005 [acesso em 2015 set 23];13(6): 944-950. Disponível em: <http://www.scielo.br/pdf/rlae/v13n6/v13n6a05.pdf>.

17. Campos EMP. A Psico-Oncologia: Uma nova visão do câncer- uma trajetória [dissertação] [internet]. São Paulo: Universidade de São Paulo; 2010 [acesso em 2015 set 21]. Disponível em: $<$ http://www.teses.usp.br/teses/disponiveis/livredocencia/47/tde-05072012-212628/ptbr.php>.

18. Magalhães MV, Melo SCA. Morte e Luto: o sofrimento do profissional da saúde. PsicSaúd Debate [periódicona internet]. 2015 [acessoem 2016 ago 17].1(1):65-77. Disponível em: <https://psicodebate.files.wordpress.com/2014/12/5-magalhc3a3esmelo-2015.pdf>.

19. Holland JC. History of psycho-Oncology: Overcoming attitudinal andconceptual barriers. Psychosomatic Medicine [periódico na internet]. 2002 [acesso em 2015 out 10].64:206-221. Disponível em: <http://www.ncbi.nlm.nih.gov/pubmed/11914437>.

20. Veit MT, Carvalho VA. Psico-Oncologia: um novo olhar para o câncer. O Mundo da Saúde[periódico na internet]. 2010[acesso em 2015 out 10]; 34(4): 526-530.Disponível em: <http://www.saocamilo-sp.br/pdf/mundo_saude/79/526a530.pdf>. 
21. Bayés R. Aspectos psicológicos do enfermo comcâncer: o impactopsicológico docâncer. InBarónMG (Org.). Tratado de Medicina Paliativa e de suporte ao enfermo comcâncer. Madrid: Panamericana; 1996.

22. Carvalho CSU, et al. A Necessária Atenção à Família do Paciente Oncológico. RevBrasCancerol[periódico na internet]. 2008 [acesso em 2015 out 5]; 54(1):87-96. Disponível <http://www.inca.gov.br/rbc/n_54/v01/pdf/revisao_7_pag_97a102.pdf>.

em:

23. Costa Junior AL. O papel da psicologia no atendimento a crianças com câncer. Brasília: Laboratório de Saúde e Desenvolvimento Humano; 2010.

24. Silva F, Bervique JA. Psico-Oncologia: lidando com a doença, o doente e a morte. RevCientEletPsicol[periódico na internet]. 2005 [acesso em 2015 set 21]; 3(5):9-10. Disponível em: <http://faef.revista.inf.br/imagens_arquivos/arquivos_destaque/ZrorgJNimECIQGP_2 013-4-30-16-28-51.pdf>.

25. Junqueira LCU. Vivências de médicos oncologistas: um estudo da religiosidade no cuidado existencial em saúde [tese]. Ribeirão Preto: Universidade de São Paulo; 2008.

26. Costa RV, Pakenham KI.Associations between benefit finding and adjustment outcomes in thyroid cancer. Psycho-Oncology[periódico na internet]. 2011[acesso em 2015 set 24]; 21(7):737-744.Disponível em: <http://www.ncbi.nlm.nih.gov/pubmed/21416554>.

27. Souza JR,Seidl EMF. Distress e enfrentamento: da teoria à prática empsico-oncologia. Brasília Med[periódico na internet]. 2014 [acesso em2015 set 21]; 50(3):242-252. Disponível em: <http://www.ambr.org.br/wp-content/uploads/2014/07/09_BSB819_Distress_V003.pdf>.

28. Stanton AL, et al. Health psychology: psychological adjustment to chronic disease. AnnualReviewofPsychology[periódico na internet]. 2007[acesso em 2015 set 21]; 58: 565-592. Disponível em: <http://www.ncbi.nlm.nih.gov/pubmed/16930096>.

29. Venâncio JL. Importância da Atuação do Psicólogo no Tratamento de Mulheres com Câncer de Mama. RevBrasCancerol[periódico na internet]. 2004 [acesso em 2015 set 21]; 50(1):55-63. Disponível em: <http://www.inca.gov.br/Rbc/n_50/v01/pdf/revisao3.pdf>. 
30. Paro D, Paro J, Ferreira D. O enfermeiro e o cuidar em Oncologia Pediátrica. ArqCiêncSaúd[periódico na internet]. 2005 [acesso em 2015 out 12]; 12(3): 151-57. Disponível em: <http://www.cienciasdasaude.famerp.br/racs_ol/vol-12-3/06\%20\%20ID132.pdf>.

31. Valle ERM, Vendrúsculo J. A Família da Criança com Câncer frente ao Diagnóstico da Doença: Encontros Iniciais com a Psicóloga. Pediatr Mod. [periódico na internet].1996 [acesso em 2015 out 12]; 32(7):117-138. Disponível em: <http://ojs.fsg.br/index.php/pesquisaextensao/article/viewFile/487-503/944>.

32. Maia SAF. Câncer e Morte: impacto sobre a família [dissertação] [internet]. Curitiba: Hospital Erasto Gaertner, Curitiba; 2005 [acesso em 2015 nov 11]. Disponível em: $<$ http://www.intercef.com.br/artigos/cancer-e-morte-o-impacto-sobre-o-paciente-e-afamilia.pdf>.

33. Scannavino CSS, et al. Psico-Oncologia:atuação do psicólogo no Hospital de Câncer de Barretos. Psicol USP [periódico na internet]. 2013 [acesso em 2015 set 21]; 24(1):35-53. Disponível em: <http://www.scielo.br/pdf/pusp/v24n1/v24n1a03.pdf>.

34. Costa Junior AL. O desenvolvimento da psico-oncologia: implicações para a pesquisa e intervenção profissional em saúde. PsicolCiencProf[periódico na internet]. 2001 [acesso em 2015 out 5]; 21(2):36-43. Disponível em: $<$ http://www.scielo.br/scielo.php?script=sci_arttext\&pid=S141498932001000200005\&lng=en\&nrm=iso\&tlng=pt>.

35. Cardoso FT. Câncer infantil: aspectos emocionais e atuação do psicólogo. Rev SBPH [periódico na internet]. 2007 [acesso em 2015 set 21]; 10(1):25-52. Disponível em: <http://pepsic.bvsalud.org/pdf/rsbph/v10n1/v10n1a04.pdf>.

36. Mantovani A, Mantovani CCP. Psico-oncologia e grupos: trabalhando vínculos em uma casa de apoio a pacientes com câncer. Rev SPAGESP [periódico na internet]. 2008 [acesso em 2015 out 5]; 9(1): 11-17. Disponível em: <http://pepsic.bvsalud.org/pdf/rspagesp/v9n1/v9n1a03.pdf>.

37. Brasil. Portaria GM/MS n 3.535, de 02 de setembro de 1998. Estabelece critérios para cadastramento de centros de atendimento em oncologia [periódico na internet]. Brasília; 1998 [acesso em 2015 out 5]. Disponível em: <http://www.saude.mg.gov.br/images/documentos/portaria_3535.pdf>.

38. Guimarães CA, Lipp MAN. Um olhar sobre o cuidador de pacientes oncológicos recebendo cuidados paliativos. Psico Teor Prat[periódico na internet]. 2011 [acesso em $2015 \quad$ out 5]; 13(2):50-62. Disponível em: <http://pepsic.bvsalud.org/pdf/ptp/v13n2/v13n2a04.pdf>. 